\title{
The Role of Diffusion Weighted MRI in Differentiation of Intra-Axial Cystic Brain Lesions
}

\author{
YASSER ABD EL AZIM, M.D.; EMAN A. FOUAD DARWISH, M.D. and AMNA A. HAYDER, M.Sc. \\ The Department of Diagnostic Radiology, Faculty of Medicine, Ain Shams University
}

\begin{abstract}
Background: Intracranial cystic lesions are unnatural cavities in which the continuity of the brain parenchyma is disrupted because of different pathologies such as infections and tumors containing proteinaceous fluid and/or cellular/necrotic materials.

Aim of Study: The purpose of this study is to investigate the value of DWI in differentiation of the common intracranial intra-axial cystic/necrotic lesions.

Patients and Methods: This is a descriptive study, conducted from July 2019 till February 2021. It is included twenty-four adult patients of either sex with a cystic/necrotic intra-axial brain lesion with variable perifocal edema and rim enhancement on post-contrast study at MRI unit, radiology department, Ain Shams University (ASU) Hospital. All examinations carried out after signing the informed consent by the patient himself or his guardian if the patient is incapacitated by any means.
\end{abstract}

Results: The inflammatory lesions, the wall ADC values were not uniform. We found the lowest ADC value in contrast enhanced part of the pyogenic abscess $0.922 \times 10^{-3} \mathrm{~mm}^{2} / \mathrm{s}$ and the highest ADC wall value $1.529 \times 10^{-3} \mathrm{~mm}^{2} / \mathrm{s}$ found in toxoplasmosis abscess. There was a significant difference in the wall ADC values of metastatic lesions mean ADC of contrast enhanced part was $1.32 \pm 0.18 \times 10^{-3} \mathrm{~mm}^{2} / \mathrm{s}$ and 0.80 $\pm 0.13 \times 10^{-3} \mathrm{~mm}^{2} / \mathrm{s}$ found in PCNSL, with a cut off value, sensitivity, specificity, positive predictive value (PPV) negative predictive value (NPV) and $p$-value of: $1.035,100 \%, 100 \%$, $100 \%, 100 \%$ and 0.007 respectively. The mean ADC value of the surrounding edema in our study were measured 1.40 $\pm 0.11 \times 10^{-3} \mathrm{~mm}^{2} / \mathrm{s}$ for abscess group and $1.59 \pm 0.31 \times 10^{-3}$ $\mathrm{mm}^{2} / \mathrm{s}$ for malignant group with insignificant $p$-value of 0.201 .

Conclusion: Further more MR techniques may be used for discrimination brain abscess from cystic or necrotic tumors, as diffusion tensor imaging (DTI), MR spectroscopy and perfusion weighted imaging as well as positron emission tomography computed tomography (PET-CT) may be helpful. The use of these additional techniques will help to improve the accuracy of MR in differentiating brain abscess from cystic or necrotic tumors.

Key Words: Diffusion Weighted MRI - Differentiation - Intraaxial cystic - Brain lesions.

Correspondence to: Dr. Amna A. Hayder, E-Mail: amna.hayder86@yahoo.com

\section{Introduction}

INTRACRANIAL cystic lesions are unnatural cavities in which the continuity of the brain parenchyma is disrupted because of different pathologies such as infections and tumors containing proteinaceous fluid and/or cellular/necrotic materials [1]

The differential diagnosis of cystic ring enhancing cerebral lesions includes, most frequently, highgrade gliomas, malignant lymphomas, metastases, and abscesses, Because of the different therapies for all these diseases, the possibility of their reliable differentiation with MRI is highly desirable [2] .

Differentiation between cerebral abscesses in capsular stage and intra-cerebral cystic or necrotic tumors is a diagnostic challenge to clinicians and radiologists because the presenting clinical manifestations are non specific On conventional MRI, the abscess center usually shows hypointensity relative to the brain parenchyma on $\mathrm{T} 1$ weighted image. The abscess wall usually shows a ring of enhancement after contrast administration. However, these findings can also be seen in necrotic or cystic brain tumors. Although, marked hypointensity of the abscess capsule as seen on T2 weighted MRI is a characteristic of abscess that allows the differentiation between infection and tumor, the hypointense capsule is not always seen in all abscesses and may be seen in some tumors. Thus, conventional MR imaging cannot reliably differentiate between these two entities most of the time leading to diagnostic dilemma [3].

Pretreatment characterization and differentiation of malignant brain tumors using MR imaging is still a challenging problem in every day practice The critical clinical problem is differentiation between high grade gliomas, metastases and primary central nervous system lymphomas (PCNSLs) 
as management can differ substantially, depending on the type of a lesion. The standard treatment of GBMs and metastases consists of surgical resection, radiotherapy, and chemotherapy while PCNSLs should not undergo surgical management but only chemotherapy [4]

On conventional magnetic resonance imaging (MRI) the appearance of a high grade glioma and a necrotic solitary metastasis is usually a ringshaped contrast-enhanced mass lesion with central hypointense necrosis on contrast-enhanced T1weighted MR imaging ( $\mathrm{T} 1 \mathrm{WI}$ ), whereas primary cerebral lymphoma (PCL) usually presenst as a solid mass lesion with homogeneous contrast enhancement in immunocompetent patients. However, this pattern is not reliable in some clinical scenarios where an atypical PCL with visible necrosis may mimic the appearance of necrotic high grade gliomas and metastasis [5]

Diffusion MR imaging is a technique in which dedicated phase-defocusing and -refocusing gradients allow evaluation of microscopic water diffusion within tissues (Brownian motion) which can be quantitated in terms of apparent diffusion coefficient (ADC). It has been found to have useful clinical applications in diagnosing and treating ischemia, tumors, epilepsy, and white matter disorders. Additionally many publications have addressed the value of DWI and in the differential diagnosis of abscess and cystic or necrotic brain tumors by virtue of restricted and facilitated diffusion in the central core, respectively [6].

Furthermore assessment of the necrotic core, the most strongly-enhanced tumor area, and the peritumoral edema with DWI and ADC has shown promising results in differentiation of high grade gliomas, solitary brain metastasis and atypical PCNSL [4,5]

\section{Aim of the work:}

The purpose of this study is to investigate the value of DWI in differentiation of the common intracranial intra-axial cystic/necrotic lesions.

\section{Patients and Methods}

\section{Patients:}

This is a descriptive study, conducted from July 2019 till February 2021. It is included twenty-four adult patients of either sex with a cystic/necrotic intra-axial brain lesion with variable perifocal edema and rim enhancement on postcontrast study at MRI unit, radiology department, Ain Shams University (ASU) Hospital. All examinations carried out after signing the informed consent by the patient himself or his guardian if the patient is incapacitated by any means.

\section{Patient preparation:}

- Full history will be taken from all patients.

- Ensuring adequate renal function via calculation of GFR from recent serum creatinine level.

- Ensuring venous access via insertion of IV cannula suitable for contrast administration.

\section{Inclusion criteria:}

Adult patients of either sex with a cystic / necrotic intra-axial brain lesion with variable perifocal edema and rim enhancement on postcontrast study.

\section{Exclusion criteria:}

- Patients known to have contraindications for MRI, e.g. an implanted magnetic device and pacemakers.

- Patients with contraindication to contrast media e.g. elevated renal functions.

- Lesions with large area of susceptability thought to be related to calcifications and/or hemorrhage.

- Patients below 18 years of age.

\section{Technique and MR imaging data acquisition:}

\section{Conventional MRI:}

The study will be performed on 1.5 Tesla MRI machine using head coil with patient lying in the supine position.

All patients will undergo routine MR brain imaging, including:

- Sagittal and axial T1-wieghted non-contrast images with imaging parameters of 538/10 (TR/TE).

- Axial fast spin-echo T2-weighted, T2-weighted spin-echo images with imaging parameters of 4130/120 (TR/TE).

- Axial fluid-attenuated inversion recovery (FLAIR) with imaging parameters of 11000/140 (TR/TE).

- Post contrast enhanced axial, coronal, and sagittal T1-weighted images.

\section{Diffusion weighted MR imaging (DWI):}

The imaging sequence for DWI will be a multi_section single shot spin echo EPI sequence (TR/TE/NEX) 3690/115ms with diffusion sensitivities of $b$-value $=0$ and $1000 \mathrm{~s} / \mathrm{mm}^{2}$. The diffusion gradients will be applied sequentially in three orthogonal directions (X,Y, \& Z). Sections of $5 \mathrm{~mm}$ thickness, interstice gap of $1 \mathrm{~mm}$, FOV 220x240mm and matrix of 256x256 will be used for all images. The ADC map will be generated automatically by MRI software. 


\section{Image analysis and results:}

Conventional MR sequences including post contrast images will be used to localize the lesion and identify three lesional subsites for further analysis namely the central cystic content, solid enhancing portion and the surrounding edema. Once identified, for each lesion, each of these areas will be qualitatively assessed for presence or absence of restricted diffusion depending on the signal intensity on the DWI and corresponding ADC map. Restricted diffusion is defined as an area of high signal intensity on b 1000 image and a corresponding dark area on the ADC map. For quantitative analysis at least 3 different regions of interest will be placed within each of the three sub sites described above on consecutive slices on the ADC map for every lesion assessed. If any of these sub sites are small, ROIs will be placed in the sub site analysed within the same slice but care will be taken to ensure that the ROIs don't overlap. The $\mathrm{ADC}$ value for each ROI will be obtained and then averaged so that at the end of the analysis there will be three mean ADC values, one for each of the three lesional subsites. Finally for each patient ADC Measurements will be made in the contralateral normal white matter and in the CSF of the lateral ventricle and ratios of average $\mathrm{ADC}$ of each subsite to the contralateral white matter and CSF will be computed for each lesion. The definitive diagnosis of the lesions will be determined by hisopathological evaluation of tissue samples taken from the different lesions either via stereotactic biopsy of postoperatively.

\section{Statistical analysis:}

The data were analyzed using Statistical Package for Social Science (IBM Corp, released 2013. IBM SPSS statistics for windows, V. 22.0. Armonk, NY. USA). Parametric quantitative data were expressed as mean \pm standard deviation (SD). Qualitative data were described as frequency and percentage. All $p$-values were two-tailed and $p$-value $<0.05$ was considered statistically significant.

\section{Results}

Twenty-four patients (14 males and 10 females) were included in the current study. The age range was 30 to 77 years of whole study population. No significant difference was found between the mean patient ages among all SOL groups $(p=0.872)$, the mean patient age in metastasis, lymphoma, GBM and abscess $53.13 \pm 11.67,52 \pm 1.63,49.14 \pm 9.65$ and $49.2 \pm 13.9$ respectively.

There was a significant association between DWI signal intensity in the lesion wall and the nature of the SOL $(p=0.006)$, where a significant larger proportion of malignant lesion $(73.7 \%)$ exhibited hyperintensity in DWI, on the other hand, DWI isointense signal was noted in all abscess lesion.

A significant association was noted between the ADC signal intensity in the lesion center and the nature of the SOL $(p=0.042)$, as the percent of malignant lesion $(89.5 \%)$ exhibiting high signal in its center is significantly higher than the abscess group percent, on the other hand ADC hypointense signal was noted in $60 \%$ of abscess lesions.

The brain SOL either abscess or malignant, could be differentiated using the mean and the normalized ADC values in the center of SOL. Abscess group showed statistically significantly lower mean ADC values $(p=0.034)$ and normalized ADC values $(p=0.025)$.

The Receiver operating characteristic (ROC) curve analysis of ADC and NADC in the SOL center revealed that the optimal cut off value for discrimination between center of all malignant SOL and abscess lesions were 1.755 and 2.3665, respectively.

The size of SOL was statistically significant different among all SOL $(\mathrm{F}=3.784, p=0.027)$. The post hoc test revealed that the mean value of GBM size $(2.068 \pm 1.344)$ was significantly higher than that of metastasis $(2.068 \pm 0.6)$.

A significant association was noted between the DWI signal intensity in the lesion center and the nature of the SOL $(p=<0.001)$, as low DWI signal in SOL center was seen in $100 \%$ of GBM and $75 \%$ of metastasis, while a hyperintense signal was seen in $60 \%$ of abscess lesions. The center of all lymphoma SOL exhibited isointense signal in the DWI.

A significant association was also noted between the DWI signal intensity in the lesion wall and the nature of the SOL ( $p=0.006)$, as high DWI signal was seen in all lymphoma SOL, $85.7 \%$ of GBM and $50 \%$ of metastasis, while all abscess SOL exhibited isointense signal in the DWI along their wall.

A significant association was also noted between the ADC signal intensity in the lesion wall and the nature of the SOL $(p=<0.001)$, where isointense ADC signal was noted in all metastasis SOL, $85.7 \%$ of GBM and $80 \%$ of abscess, while all lymphoma SOL exhibited hypointense signal in the ADC along their wall. 
The perilesional edema revealed hyperintense signal in the ADC map in all SOL.

The mean ADC value of SOL center was statistically significant different among all SOL $(\mathrm{F}=3.969, p=0.023)$. The post hoc test revealed that the mean ADC value of GBM $(2.71 \pm 0.29)$ was significantly higher than that of lymphoma $(1.78 \pm 0.02)$.

The mean ADC value of SOL wall was statistically significant different among all $\mathrm{SOL}(\mathrm{F}=$ $4.026, p=0.022)$. The post hoc test revealed that the mean ADC value of metastasis $(1.32 \pm 0.18)$ was significantly higher than that of lymphoma $(0.8 \pm 0.13)$.
The mean NADC value of SOL center was statistically significant different among all SOL $(\mathrm{F}=4.892$, $p=0.01$ ). The post hoc test revealed that the mean ADC value of GBM $(3.56 \pm 0.36)$ was significantly higher than that of lymphoma $(2.43 \pm 0.04)$.

The Receiver operating characteristic (ROC) curve analysis of ADC and NADC in the SOL center revealed that the optimal cut off values for discrimination between center of GBM and lymphoma were 1.755 and 2.3665 , respectively.

The Receiver operating characteristic (ROC) curve analysis of ADC in the wall of SOL revealed that the optimal cut off value for discrimination between wall of metastasis and lymphoma was 1.035 .

Table (1): Description of patients' age among all SOL lesions.

\begin{tabular}{llllcccccc}
\hline & Mean & SD & Median & Min & Max & IQR & F & $p$-value & Sig. \\
\hline Abscess & 49.20 & 13.9 & 50 & 30 & 68 & 24 & 0.234 & 0.872 & NS \\
GBM & 49.14 & 9.65 & 51 & 33 & 58 & 17 & & & \\
Mets & 53.13 & 11.67 & 53.5 & 39 & 77 & 12 & & & \\
Lymphoma & 52 & 1.63 & 52 & 50 & 54 & 3 & & & \\
\hline
\end{tabular}

$\mathrm{F}$ test $=$ ANOVA. $\quad$ NS $=$ Non-significant.

Table (2): Description of patients' sex among all SOL lesions.

\begin{tabular}{|c|c|c|c|c|c|c|c|c|c|c|c|}
\hline & \multicolumn{2}{|c|}{ Abscess } & \multicolumn{2}{|c|}{ GBM } & \multicolumn{2}{|c|}{ Mets } & \multicolumn{2}{|c|}{ Lymphoma } & \multicolumn{3}{|c|}{ Fisher's Exact Test } \\
\hline & No & Percent & No & Percent & No & Percent & No & Percent & Value & $p$-value & Sig. \\
\hline \multicolumn{12}{|l|}{ Sex: } \\
\hline Female & 2 & 40 & 2 & 28.6 & 2 & 25 & 4 & 100 & 6.506 & 0.085 & NS \\
\hline Male & 3 & 60 & 5 & 71.4 & 6 & 75 & 0 & 0 & & & \\
\hline
\end{tabular}

Table (3): Distribution of qualitative DWI signal intensity in the lesion wall (W DWI) among abscess and all malignant SOL.

\begin{tabular}{|c|c|c|c|c|c|c|c|}
\hline & \multicolumn{2}{|c|}{ Abscess } & \multicolumn{2}{|c|}{ Malignant } & \multicolumn{3}{|c|}{ Fisher's Exact Test } \\
\hline & No & $(\%)$ & No & $(\%)$ & Value & $p$-value & Sig. \\
\hline Hypointense & 0 & 0 & 1 & 5.3 & 9.913 & 0.006 & S \\
\hline Isointense & 5 & 100 & 4 & 21.1 & & & \\
\hline Hyperintense & 0 & 0 & 14 & 73.7 & & & \\
\hline
\end{tabular}

S = Significant NB: $66.7 \%$ of cells have expected count less than 5, so the test is week due to small sample size.

Table (4): Distribution of qualitative ADC signal intense in the lesion center (C ADC) among abscess and all malignant lesions.

\begin{tabular}{|c|c|c|c|c|c|c|c|}
\hline & \multicolumn{2}{|c|}{ Abscess } & \multicolumn{2}{|c|}{ Malignant } & \multicolumn{3}{|c|}{ Fisher's Exact Test } \\
\hline & No & $(\%)$ & No & $(\%)$ & Value & $p$-value & Sig. \\
\hline Hypointense & 3 & 60 & 2 & 10.5 & 5.874 & 0.042 & S \\
\hline Isointense & 0 & 0 & 0 & 0 & & & \\
\hline Hyperintense & 2 & 40 & 17 & 89.5 & & & \\
\hline
\end{tabular}

$\mathrm{S}=$ Significant NB: $75 \%$ of cells have expected count less than 5 , so the test is week due to small sample size. 
Table (5): Description of quantitative ADC and NADC value among abscess and all malignant lesions in the lesion center (C ADC \& C NADC).

\begin{tabular}{lcccccccccc}
\hline & Group & Mean & SD & Median & Min & Max & IQR & Value* & $p$-value & Sig. \\
\hline C ADC QT Abscess & 1.45 & 0.83 & 0.91 & 0.89 & 2.79 & 1.37 & -2.267 & 0.034 & $\mathrm{~S}$ \\
& Malignant & 2.26 & 0.68 & 2.64 & 0.84 & 3.07 & 1.08 & & \\
\multirow{2}{*}{ C NADC } & Abscess & 1.87 & 1.09 & 1.22 & 1.10 & 3.58 & 1.86 & -2.402 & 0.025 & $\mathrm{~S}$ \\
& Malignant & 2.93 & 0.82 & 3.30 & 1.02 & 3.98 & 1.11 & & \\
\hline
\end{tabular}

$\mathrm{IQR}=$ Interquartile range. $\quad *=$ Independent sample $t$-test.

Table (6): The cut off value, sensitivities, specificities, positive predictive values (PPV) and negative predictive values (NPV) of the cut off values of the ADC and NADC in SOL center for the differentiation of the abscess from malignant group.

\begin{tabular}{lccccccccc}
\hline & Cut off value+ & Sensitivity & Specificity & PPV & NPV & Accuracy & AUC & $p$-value & $95 \%$ CI \\
\hline C ADC QT & 1.755 & $84.2 \%$ & $80 \%$ & $94.1 \%$ & $57.1 \%$ & $83.3 \%$ & 0.789 & $<0.051^{*}$ & $0.532-1.000$ \\
C NADC & 2.3665 & $89.5 \%$ & $80 \%$ & $94.4 \%$ & $66.7 \%$ & $87.5 \%$ & 0.789 & $0.051^{*}$ & $0.532-1.000$ \\
\hline
\end{tabular}

AUC $=$ Area under curve. $*$ Marginally Significant. + Value greater than cut off value denoting malignant SOL.

Table (7): Descriptive data of different SOL size.

\begin{tabular}{lllllllllr}
\hline SOL & Mean & SD & Median & Min & Max & IQR & F & $p$-value & Sig \\
\hline Abscess & 2.018 & 0.485 & 1.9 & 1.58 & 2.84 & 0.74 & 3.784 & 0.027 & S \\
GBM & 3.4 & 1.344 & 3.12 & 1.93 & 6.20 & 0.76 & & & \\
Mets & 2.068 & 0.6 & 1.9 & 1.44 & 3.03 & 1.15 & & & \\
Lymphoma & 2.892 & 0.614 & 3.105 & 2.00 & 3.36 & 1.08 & & & \\
\hline
\end{tabular}

$\mathrm{F}$ test $=$ ANOVA.

Table (8): Significant Post hoc test* between GBM and Mets size.

\begin{tabular}{ccccc}
\hline 1 st SOL & 2nd SOL & Mean Difference & $p$-value & Sig \\
\hline GBM & Mets & 1.331 & .038 & S \\
\hline
\end{tabular}

*Tukey post hoc test.

Table (9): Distribution of qualitative C DWI signal intensity among all SOL lesions.

\begin{tabular}{|c|c|c|c|c|c|c|c|c|c|c|c|}
\hline & \multicolumn{2}{|c|}{ Abscess } & \multicolumn{2}{|c|}{ GBM } & \multicolumn{2}{|c|}{ Mets } & \multicolumn{2}{|c|}{ Lymphoma } & \multicolumn{3}{|c|}{ Fisher's Exact Test } \\
\hline & No & $(\%)$ & No & $(\%)$ & No & $(\%)$ & No & $(\%)$ & Value & $p$-value & Sig \\
\hline Hypointense & 2 & 40 & 7 & 100 & 6 & 75 & 0 & 0 & 19.348 & $<0.001$ & $\mathrm{~S}$ \\
\hline Isointense & 0 & 0 & 0 & 0 & 0 & 0 & 4 & 100 & & & \\
\hline Hyperintense & 3 & 60 & 0 & 0 & 2 & 25 & 0 & 0 & & & \\
\hline
\end{tabular}

$\mathrm{S}=$ Significant

NB: $91.7 \%$ of cells have expected count less than 5 , so the test is week due to small sample size.

Table (10): Distribution of qualitative W DWI signal intensity among all SOL lesions.

\begin{tabular}{|c|c|c|c|c|c|c|c|c|c|c|c|}
\hline & \multicolumn{2}{|c|}{ Abscess } & \multicolumn{2}{|c|}{ GBM } & \multicolumn{2}{|c|}{ Mets } & \multicolumn{2}{|c|}{ Lymphoma } & \multicolumn{3}{|c|}{ Fisher's Exact Test } \\
\hline & No & $(\%)$ & No & $(\%)$ & No & $(\%)$ & No & $(\%)$ & Value & $p$-value & Sig \\
\hline Hypointense & 0 & 0 & 0 & 0 & 1 & 12.5 & 0 & 0 & 13.802 & 0.006 & S \\
\hline Isointense & 5 & 100 & 1 & 14.3 & 3 & 37.5 & 0 & 0 & & & \\
\hline Hyperintense & 0 & 0 & 6 & 85.7 & 4 & 50 & 4 & 100 & & & \\
\hline
\end{tabular}

$\mathrm{S}=$ Significant

NB: $100 \%$ of cells have expected count less than 5 , so the test is week due to small sample size. 
Table (11): Distribution of qualitative W ADC signal intensity among all SOL lesions.

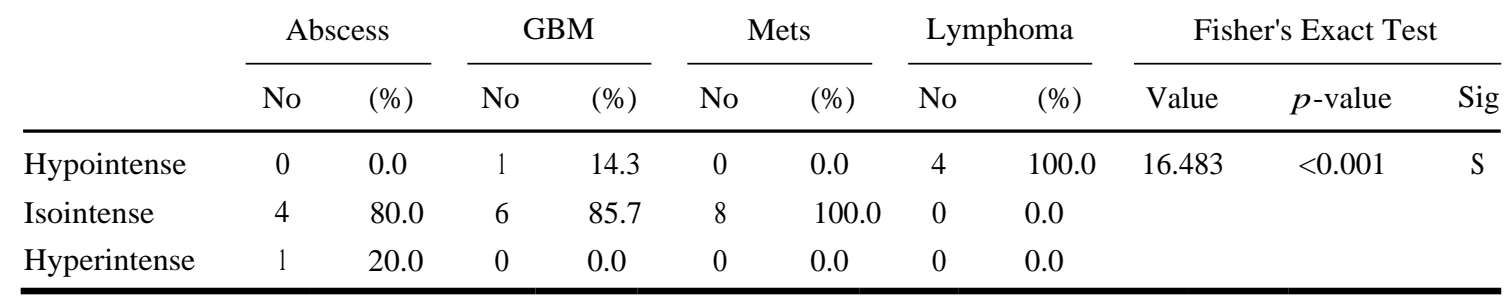

$\mathrm{S}=$ Significant

NB: $83.3 \%$ of cells have expected count less than 5 , so the test is week due to small sample size.

Table (12): Distribution of qualitative E ADC signal intensity among all SOL lesions.

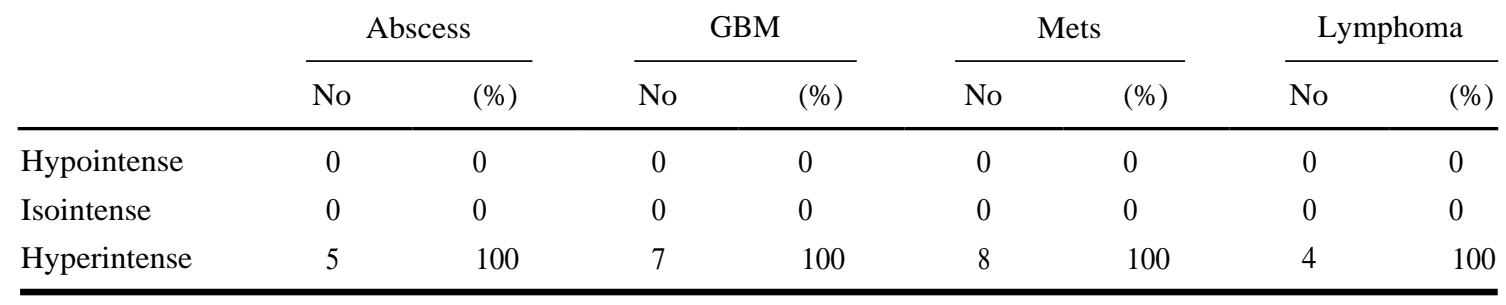

NB: Fisher's Exact Test can't be computed.

Table (13): Description of C ADC value among all SOL lesions.

\begin{tabular}{lccccccccc} 
& Mean & SD & Median & Min & Max & IQR & F & $p$-value & Sig \\
\hline Abscess & 1.45 & 0.83 & 0.91 & 0.89 & 2.79 & 1.37 & 3.969 & 0.023 & S \\
GBM & 2.71 & 0.29 & 2.76 & 2.11 & 3.05 & 0.22 & & & \\
Mets & 2.12 & 0.87 & 2.38 & 0.84 & 3.07 & 1.68 & & & \\
Lymphoma & 1.78 & 0.02 & 1.78 & 1.76 & 1.80 & 0.04 & & & \\
\hline
\end{tabular}

F test $=$ ANOVA.

Table (14): Significant Post hoc test* between GBM and lymphoma.

\begin{tabular}{lcccc}
1 st SOL & 2nd SOL & Mean Difference & $p$-value & Sig \\
\hline GBM & Lymphoma & 0.935 & 0.001 & $\mathrm{~S}$ \\
\hline
\end{tabular}

* Games-Howell post hoc (inhomogeneous variance).

Table (15): Description of W ADC value among all SOL lesions.

\begin{tabular}{lccccccccc}
\hline & Mean & SD & Median & Min & Max & IQR & F & $p$-value & Sig \\
\hline Abscess & 1.18 & 0.23 & 1.15 & 0.92 & 1.53 & 0.39 & 4.026 & 0.022 & $\mathrm{~S}$ \\
GBM & 1.15 & 0.35 & 1.23 & 0.41 & 1.46 & 0.25 & & & \\
Mets & 1.32 & 0.18 & 1.27 & 1.16 & 1.63 & 0.32 & & & \\
Lymphoma & 0.80 & 0.13 & 0.80 & 0.69 & 0.92 & 0.23 & & & \\
\hline
\end{tabular}

$\mathrm{F}$ test $=$ ANOVA.

Table (16): Significant Post hoc test* between Mets and lymphoma.

\begin{tabular}{lcccc} 
1 st SOL & 2nd SOL & Mean Difference & $p$-value & Sig \\
\hline Mets & Lymphoma & 0.519 & 0.012 & $\mathrm{~S}$ \\
\hline
\end{tabular}

*Tukey post hoc test. 
Table (17): Description of C NADC value among all SOL lesions.

\begin{tabular}{lccccccccr}
\hline & Mean & SD & Median & Min & Max & IQR & F & $p$-value & Sig \\
\hline Abscess & 1.87 & 1.09 & 1.22 & 1.10 & 3.58 & 1.86 & 4.892 & 0.01 & S \\
GBM & 3.56 & 0.36 & 3.67 & 2.90 & 3.98 & 0.55 & & & \\
Mets & 2.62 & 0.98 & 2.93 & 1.02 & 3.51 & 1.83 & & & \\
Lymphoma & 2.43 & 0.04 & 2.43 & 2.39 & 2.47 & 0.07 & & & \\
\hline
\end{tabular}

$\mathrm{F}$ test $=$ ANOVA

Table (18): Significant Post hoc test* between GBM and lymphoma.

\begin{tabular}{lcccc}
\hline 1 st SOL & 2nd SOL & Mean Difference & $p$-value & Sig \\
\hline GBM & Lymphoma & 1.1336 & 0.001 & $\mathrm{~S}$ \\
\hline
\end{tabular}

* Games-Howell post hoc test.

Table (19): The cut off value, sensitivities, specificities, positive predictive values (PPV) and negative predictive values (NPV) of the cut off values of the ADC and NADC in SOL center for the differentiation of GBM and lymphoma.

\begin{tabular}{lccccccccc}
\hline & Cut off value+ & Sensitivity & Specificity & PPV & NPV & Accuracy & AUC & $p$-value & $95 \%$ CI \\
\hline C ADC & 1.955 & $100 \%$ & $100 \%$ & $100 \%$ & $100 \%$ & $100 \%$ & 1 & $0.008^{*}$ & $1-1$ \\
C NADC & 2.682 & $100 \%$ & $100 \%$ & $100 \%$ & $100 \%$ & $100 \%$ & 1 & $0.008^{*}$ & $1-1$ \\
\hline
\end{tabular}

AUC $=$ Area under curve. $*$ Significant + Value greater than cut off value denoting GBM.

Table (20): The cut off value, sensitivity, specificity, positive predictive value (PPV) and negative predictive value (NPV) of the SOL wall ADC cut off value for the differentiation of metastasis and lymphoma.

\begin{tabular}{cccccccccc}
\hline & Cut off value+ & Sensitivity & Specificity & PPV & NPV & Accuracy & AUC & $p$-value & $95 \%$ CI \\
\hline W ADC & 1.035 & $100 \%$ & $100 \%$ & $100 \%$ & $100 \%$ & $100 \%$ & 1 & $0.007 *$ & $1-1$ \\
\hline
\end{tabular}

AUC $=$ area under curve. $*$ Significant. + Value greater than cut off value denoting Mets .

Illustrative Cases
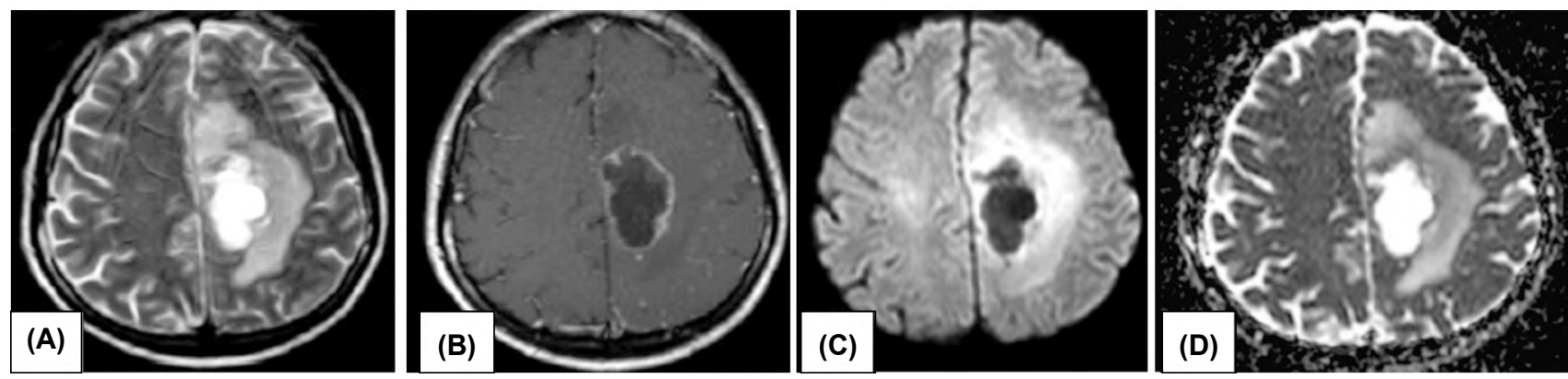

Fig. (1): 58 years old male patient complains of chronic headache. A: Axial T2- weighted shows hyperintense SOL in left high frontal parafalcin area, surrounded by marked vasogenic edema (B) Axial T1WI post-Gd revealed cystic irregular circumferential rim enhancement. C: Axial DWI revealed a hypointense signal of the central part consisted with facilitated diffusion, hyperintensity on both wall and perifocal edema. D: Axial ADC map revealed a hyperintense signal in central portion of lesion with mean ADC value $=2.726 \times 10-3 \mathrm{~mm}^{2} / \mathrm{s}$. A hypointense signal in enhancing wall with mean ADC value $=1.241$ $\times 10^{-3} \mathrm{~mm}^{2} / \mathrm{s}$, and hyper intense signal of perifocal edema with mean ADC value of $=1.714 \times 10^{-3} \mathrm{~mm}^{2} / \mathrm{s}$.

Diagnosis: Histologically proven Glioblastoma. 

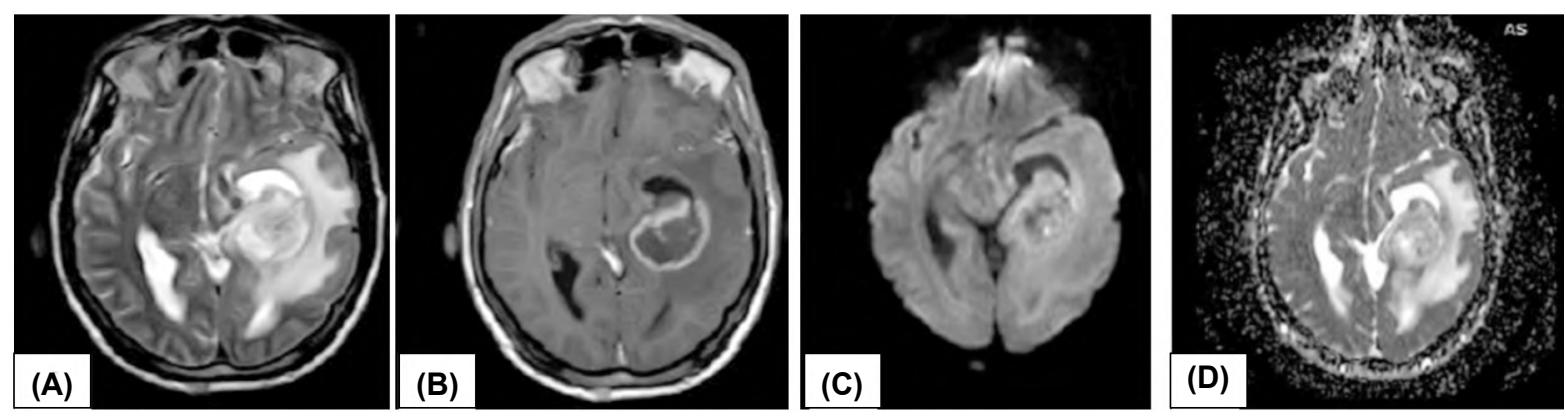

Fig. (2): 57 years old male patient complaining of headache and vomiting. (A): Axial T2- weighted exhibits high signal intensity SOL in the left temporoparietal lobe, surrounded by large amount of vasogenic edema with subsequent mass effect in form of compression of the ipsilateral occipital horn of lateral ventricle and shift of midline to the right side. (B) Axial T 1WI post-Gd shows large irregular ring enhancing lesion. (C): Axial DWI revealed a low signal intensity of the center part, consistent with facilitated diffusion and restricted diffusion signal intensity on both wall and perifocal edema. D: Axial ADC map revealed a hyperintense signal in central portion of lesion with mean ADC value $=2.111 \times 10^{-3} \mathrm{~mm}^{2} / \mathrm{s}$, consistent with lack of diffusion restriction. An isointense signal in enhancing wall with mean ADC value $=1.233 \times 10^{-3} \mathrm{~mm}^{2} / \mathrm{s}$, and hyper intense signal of perifocal edema with mean ADC value of $=1.733 \times 10^{-3} \mathrm{~mm}^{2} / \mathrm{s}$

Diagnosis: Histologically proven Glioblastoma.

\section{Discussion}

Pretreatment characterization and differentiation of malignant brain tumors using MR imaging is still a challenging problem in every day practice. The proper initial diagnosis and subsequently adequate treatment significantly influence patients surveillance but the management can differ substantially, depending on the type of a lesion. The important clinical problem is differentiation between glioblastoma multiforme (GBM), metastases, primary central nervous system lymphomas (PCNSLs) and pyogenic brain absceses which may show very similar appearance on conventional MR sequences as solitary strongly rim enhancing brain tumors surrounded by a T2-hyperintense edema. The standard treatment of GBMs and metastases consists of surgical resection, radiotherapy, and chemotherapy while PCNSLs should not undergo a surgical management but only chemotherapy [7]

While neurosurgical biopsy, aspiration and drainage either stereotactic, ultrasound guided or free hand is considered to be the first-line treatment of cerebral abscess, because expected brain tissue damage will be less compared to open surgery with abscess excision. Differentiation between cystic brain masses is important not only in diagnosis and follow-up of antibiotic therapy but also will influence neurosurgical approach to these lesions. In case of an abscess, the center should be target with intentions to aspirate pus and to culture the causing pathogens. In contrast, the optimal site for tissue diagnosis of malignant cystic tumors is the contrast enhancing tumor rim $[\mathbf{8 , 9 ]}$.

The main role of conventional MRI in brain tumors is to distinguish it's location, extent, mor- phology, and relation to important structures, but alone it is deficient in diagnostic examination. Thus, there is a need for additional imaging modalities such as Diffusion weighted image (DWI) which may aid in diagnosis and differentiation of brain tumors. DWI provides image contrast based on differences in the diffusion characteristics of water molecules within brain tissue. The magnitude of diffusion can be quantified by calculation of the apparent diffusion coefficient (ADC). Diffusion of water molecules within brain tissue is determined by the microstructure in which the diffusion occurs [10].

Accordingly on diffusion weighted sequences, the difference observed in signal intensities between abscess and cystic/necrotic tumors is mainly due to differences in the consistency and viscosity of central content of lesions. In abscess, the contents consist of thick viscous pus containing inflammatory cells, bacteria and thick proteinaceous material which restricts free Braownian movement of water molecules thus causing diffusion restriction (high intensity on DWI and low ADC value). While in case of cystic/necrotic tumors, the cavities contain thin necrotic debris, more serous fluid and few inflammatory cells as compared to abscess thus causing free diffusion (low intensity on DWI, high ADC value) [11] .

Differentiation of ring-enhanced cerebral lesions includes high grade gliomas, malignant lymphomas, metastases, pyogenic abscess, and in rare cases demyelinating disease or sub-acute ischemic infarctions and resolving hematomas, can help the neurosurgeon to determine the most appropriate management. 
Several of these previous reports suggested that the necrotic parts of tumorous lesions show low signal on DWI, with corresponding high ADC values, whereas the central parts of abscesses give high signal on DWI, showing restricted diffusion and low ADCs.

Decreased diffusion was shown to be characteristic of non-abscess SOL (malignant tumors). The results of this study supported these facts where both absolute ADC values and normalized ADC values of central part of lesions were significantly higher in necrotic parts of malignant tumours $\left(2.26 \pm 0.68 \times 10^{-3} \mathrm{~mm}^{2} / \mathrm{s}\right.$ and $2.93 \pm 0.82 \times 10^{-3}$ $\mathrm{mm}^{2} / \mathrm{s}$ respectively) than central parts of abscess $\left(1.45 \pm 0.83 \times 10^{-3} \mathrm{~mm}^{2} / \mathrm{s}\right.$ and $1.87 \pm 1.09 \times 10^{-3} \mathrm{~mm}^{2} / \mathrm{s}$ for absolute center ADC and normalized ADC respectively). A cutoff ADC value and NADC in the SOL center revealed that the optimal cut off value for discrimination between center of all malignant SOL and abscess lesions were 1.755 $\times 10^{-9} \mathrm{~mm}^{2} / \mathrm{s}$ and $2.3665 \times 10^{-3} \mathrm{~mm}^{2} / \mathrm{s}$, respectively. Similar results were demonstrated by several previous studies including that done by Desbarats et al. whose results confirmed that ADC values are significantly higher in malignant center to abscess center. Likewise they found a cutoff ADC value of $>1.10 \times 10^{-3} \mathrm{~mm}^{2} / \mathrm{s}$ accurate for diagnosing necrotic/cystic tumors [12]

In the present study, 3 of 5 (60\%) patients with brain abscesses showed in their central/necrotic parts restricted diffusion with hyperintensity on DWI and hypointensity on ADC map, with sensitivity of $84.2 \%$ and specificity of $80 \%$ in differentiating it from non-abscesses. Whereas 2 patients with brain abscesses presented facilated diffusion with hypointensity on DWI and hyperintensity on ADC map. One of these facilitated DWI was fungal abscess (central cystic part ADC of 2.79) and the other one was toxoplasmosis brain abscess (central necrotic portion ADC of 1.753). This was consistent with the results of several previous studies $[\mathbf{1 , 1 0 -}$ 15].

Most of the previous studies found significant difference between the ADC values of patients with brain abscess and cystic tumors. Reddy et al., found the ADC of the abscess group to be $0.87 \pm$ $0.05 \times 10^{-3} \mathrm{~mm}^{2} / \mathrm{s}$ and was significantly low compared with the non-abscess group $\left(2.89 \pm 0.05 \times 10^{-3}\right.$ $\mathrm{mm}^{2} / \mathrm{s}$ ) [1]. Lai et al., found ADC values of 20 out 21 cases with brain abscesses to be in the range of $0.41-0.87 \times 10^{-3} \mathrm{~mm}^{2} / \mathrm{s}$ while the ADC values for 22 cases with cystic or necrotic tumors were in the range of $2.12-3.14 \times 10^{-3} \mathrm{~mm}^{2} / \mathrm{s}$, while one case of tumor showed low ADC value [14]. Also Reiche et al., reported the ADC value of brain abscess in the range of $0.39-0.67 \times 10^{-3} \mathrm{~mm}^{2} / \mathrm{s}$ while the tumors were in the range of $1.84-2.88 \times 10^{-3} \mathrm{~mm}^{2} / \mathrm{s}[15]$.

Most of the previous studies used threshold ADC value for discrimination of brain abscess from cystic or necrotic tumors in the range of 0.9$1.1 \times 10^{-3} \mathrm{~mm}^{2} / \mathrm{s}$. in the current study, the cutoff ADC value and NADC in the SOL center revealed that the optimal cut off value for discrimination between center of all malignant SOL and abscess lesions were $1.755 \times 10^{-3} \mathrm{~mm}^{2} / \mathrm{s}$ and $2.3665 \times 10^{-3}$ $\mathrm{mm}^{2} / \mathrm{s}$, respectively.

So, we also found significant difference between the ADC values of patients with brain abscess and cystic tumors these results are in good agreement with the finding of the previous studies.

Conversely, 17 (89.5\%) of 19 patients with marginal enhancing cystic tumors in the current study had center ADC values more than $1.755 \mathrm{x}$ $10^{-3} \mathrm{~mm}^{2} / \mathrm{s}$, whereas 2 tumors metastases had ADC values less than $1.755 \times 10^{-3} \mathrm{~mm}^{2} / \mathrm{s}(0.836-0.932$ $\mathrm{x} 10^{-3} \mathrm{~mm}^{2} / \mathrm{s}$ ).

Taj-Aldean reported 5 cases out of 19 patients with brain metastatic tumors showed hyperintensity on DWI and hypointensity on ADC while the remaining showed hypointensity on DWI and hyperintensity on ADC [10].

Holtas et al., reported a ring enhanced brain metastasis with hyperintensity on DWI and a low $\mathrm{ADC}$ value in the necrotic part of the tumor. The reason for restricted diffusion was possibly early necrosis with intracellular edema of the lesion [16]

In a study Reiche et al., two cystic tumors showed restricted diffusion with low ADC, and could not be differentiated precisely from abscesses. The histopathological findings of stereotactic biopsy and resection specimen revealed tumor coagulation necrosis in one case and large areas of necrosis in the other. Thus densely packed cell debris together with proteinaceous ingredients in tumor cysts alter diffusion in a small number of patients [15].

Our findings show low ADC values in abscesses, and these low ADC values are attributable to the presence of pus. Pus is a highly viscous, thick, mucoid fluid consisting of inflammatory cells, bacteria, proteoneous exudate and fibrinogen. Because of this high viscosity, diffusion water motion is severely curtailed. The high ADC values we find in cystic or necrotic lesions are attributable to an intra-cavity fluid that is less viscous than 
that found in abscesses. It consists of necrotic tissue debris and contains fewer inflammatory cells than abscess fluid $[2,12,14]$

Furthermore, some authors believe that reliable differentiation between high-grade gliomas, metastases and primary CNS lymphomas is possible. We found that the highest mean ADC value of necrotic part of GBM $\left(2.71 \pm 0.29 \times 10^{-3} \mathrm{~mm}^{2} / \mathrm{s}\right)$ was significantly higher than that of lymphoma $\left(1.78 \pm 0.02 \times 10^{-3} \mathrm{~mm}^{-3} / \mathrm{s}\right)$ (The cut off value, sensitivities, specificities of the ADC in SOL center for the differentiation of GBM and lymphoma was $1.955 \times 10^{-3} \mathrm{~mm}^{2} / \mathrm{s}, 100 \%, 100 \%$ respectively). The ADCs for metastases were only somewhat higher than PCNSL $\left(2.12 \pm 0.87 \times 10^{-3} \mathrm{~mm}^{2} / \mathrm{s}\right)$. Dorenbech et al., report that highest mean ADC value was $2.94 \pm 0.64 \times 10^{-3} \mathrm{~mm}^{2} / \mathrm{s}$, found in glioblastomas. For the metastases $\left(1.84 \pm 0.66 \times 10^{-3} \mathrm{~mm}^{2} / \mathrm{s}\right)$, the malignant lymphomas $\left(1.79 \pm 0.27 \times 10^{-3} \mathrm{~mm}^{2} / \mathrm{s}\right) .2$ Whereas Lai et al., shows metastases are highest ADC [13].

Mean ADC and normalized ADC value from the wall of neoplastic lesion is $1.15 \pm 0.31 \times 10^{-3}$ $\mathrm{mm}^{2} / \mathrm{second}$ and $1.47 \pm 0.39 \times 10^{-3} \mathrm{~mm}^{2} / \mathrm{s}$ respectively, compared to $1.18 \pm 0.23 \times 10^{-3} \mathrm{~mm}^{2} /$ second and $1.52 \pm 0.35 \times 10^{-3} \mathrm{~mm}^{2} / \mathrm{s}$ in abscess group with non significant $p$-value of 0.809 for mean ADC wall and non significant $p$-value of 0.804 for normalized ADC of wall. In study done by Alam et al., found that the mean ADC value from the wall of the lesions in neoplastic lesion is $1.17 \pm 0.10 \times 10^{-3}$ $\mathrm{mm}^{2} /$ second compared to $0.882 \pm 0.11 \times 10^{-3}$ $\mathrm{mm}^{2} / \mathrm{second}$ in abscess group with significant $p$ value of 0.025 [3].

In our study the results for the inflammatory lesions, the wall ADC values were not uniform. We found the lowest ADC value in contrast enhanced part of the pyogenic abscess $0.922 \times 10^{-3}$ $\mathrm{mm}^{2} / \mathrm{s}$ and the highest ADC wall value $1.529 \times 10$ ${ }^{3} \mathrm{~mm}^{2} / \mathrm{s}$ found in toxoplasmosis abscess.

Dorenbech et al., reported 5 abscess with wall ADC between 0.77 and $1.20 \times 10^{-3} \mathrm{~mm}^{2} / \mathrm{s}$ and and one toxoplasmosis abscess of $0.93 \times 10^{-3} \mathrm{~mm}^{2} / \mathrm{s}$ in it's contrast enhanced part [2].

In contrast there was a significant difference in the wall ADC values of metastatic lesions mean ADC of contrast enhanced part was $1.32 \pm 0.18 \times 10$ ${ }^{3} \mathrm{~mm}^{2} / \mathrm{s}$ and $0.80 \pm 0.13 \times 10^{-3} \mathrm{~mm}^{2} / \mathrm{s}$ found in PCNSL, with a cut off value, sensitivity, specificity, positive predictive value (PPV) negative predictive value (NPV) and $p$-value of: 1.035, 100\%, 100\%, $100 \%, 100 \%$ and 0.007 respectively.
Similar study done by Wang et al., involved 19 cases of brain metastasis and 12 cases of PCNSL Cut off value 0.85 , sensetivity 0.91 , specificity 0.56 was reported for differentiation of metastasis and lymphomas in the contrast enhanced part [17]

While Dorenbech found the highest wall ADC values in the glioblastomas $\left(1.68 \pm 0.37 \times 10^{-3} \mathrm{~mm}^{2} / \mathrm{s}\right)$ and the values for the malignant lymphomas, the metastases, and the astrocytomas differed slightly, with values between $1.25 \pm 0.26$ and $1.14 \pm 0.10 \mathrm{x}$ $10^{-3} \mathrm{~mm}^{2} / \mathrm{s}$ [2]

The mean ADC value of the surrounding edema in our study were measured $1.40 \pm 0.11 \times 10^{-3} \mathrm{~mm}^{2} / \mathrm{s}$ for abscess group and $1.59 \pm 0.31 \times 10^{-3} \mathrm{~mm}^{2} / \mathrm{s}$ for malignant group with insignificant $p$-value of 0.201 .

Alam et al., descried perifocal edema mean ADC values of $1.66 \pm 0.5 \times 10^{-3} \mathrm{~mm}^{2} / \mathrm{second}$ for abscesses and $1.49 \pm 1.1 \times 10^{-3} \mathrm{~mm}^{2} / \mathrm{second}$ for neoplastic lesions with insignificant $p$-value of 0.061 [3].

The ADCs in the surrounding T2/FLAIR hyperintense in all SOL was found to be overlapping of insignificant $p$-value of 0.087 , therefore differentiation of peritumoral edema and infiltration wasn't possible. As seen in the study by Bhandari, Desbarats and Dorenbeck [2,12,18]

There were certain limitations of this study. It was a single centre study which can result in selection bias. Sample size was relatively small and diffusion images were read in conjunction with routine MRI sequences which can lead to bias in making final diagnosis.

Brain abscess and necrotic tumours are most of the time difficult to differentiate on routine conventional imaging and prompt diagnosis is important as untreated brain abscess could be lethal. Diffusion imaging can aid in the diagnosis and further management plan so to help in improved patient care. It may alter the plan for biopsy and the need for additional investigations. Although this sequence has high sensitivity and specificity, it should be used as addition to conventional imaging and not as replacement for histopathology [14].

Further more MR techniques may be used for discrimination brain abscess from cystic or necrotic tumors, as diffusion tensor imaging (DTI), MR spectroscopy and perfusion weighted imaging as well as positron emission tomography computed tomography (PET-CT) may be helpful. The use of these additional techniques will help to improve 
the accuracy of MR in differentiating brain abscess from cystic or necrotic tumors.

\section{References}

1- REDDY J., MISHRA A., BEHARI S., HUSAIN M., GUPTA V., RASTOGI M. and GUPTA R.: The role of diffusion-weighted imaging in the differential diagnosis of intracranial cystic mass lesions: A report of 147 lesions. Surgical Neurology, 66 (3): 246-250, 2006.

2- DORENBECK U., BUTZ B., SCHLAIER J., BRETSCHNEIDER T., SCHUIERER G. and FEUERBACH S.: Diffusion-Weighted Echo-Planar MRI of the Brain with Calculated ADCs: A Useful Tool in the Differential Diagnosis of Tumor Necrosis from Abscess?. Journal of Neuroimaging, 13 (4): 330-338, 2003.

3- ALAM M., SAJJAD Z., AZEEMUDDIN M., et al.: Diffusion Weighted MR Imaging of Ring Enhancing Brain Lesions. Journal of the College of Physicians and Surgeons Pakistan, 22 (7): 428-31, 2012.

4- SUH C.H., KIM H.S., JUNG S.C., et al.: DiffusionWeighted Imaging and Diffusion Tensor Imaging for Differentiating High-Grade Glioma from Solitary Brain Metastasis: A Systematic Review and Meta-Analysis. American Journal of Neuroradiology, 39 (7): 1208-1214, 2018.

5- KO C.C., TAI M.H., LI C.F., et al.: Differentiation between Glioblastoma Multiforme and Primary Cerebral Lymphoma: Additional Benefits of Quantitative DiffusionWeighted MR Imaging. PLoS One, 11 (9), 2016.

6- JONES D.K.: Diffusion MRI: Theory, Methods, and Applications. Oxford University Press, 2011.

7- NESKA - MATUSZEWSKA M., BLADOWSKA J., SASIADEK M. and ZIMNY A.: Differentiation of glioblastoma multiforme, metastases and primary central nervous system lymphomas using multiparametric perfusion and diffusion MR imaging of a tumor core and a peritumoral zone-Searching for a practical approach. PLOS ONE, 13 (1): e0191341, 2018.

8- STROWITZKI M., SCHWERDTFEGER K. and STEUDEL W.I.: Ultrasound-guided aspiration of brain abscesses through a single burr hole. Minim Invasive Neurosurg, 44: 135-140, 2001.
9- LEUTHARDT E.C., WIPPOLD F.J., OSWOOD M.C., et al.: Diffusion-weighted MR imaging in the preoperative assessment of brain abscesses. Surg Neurol.,, 58: 395402, 2002.

10- TAJ-ALDEAN K.: Role of Diffusion-Weight MRI in Differential Diagnosis of Cerebral Cystic Lesions: A Prospective Study. International Journal of Pharmaceutical and Clinical Research, 9 (1), 2017.

11- GUL H.: Role of diffusion weighted imaging to differentiate between brain abscess and cystic/necrotic brain tumor. J. Med. Sci., 25 (1): 110-114, 2017.

12- DESBARATS N., HERLIDOU S., de MARCO G., et al.: Differential MRI diagnosis between brain abscesses and necrotic or cystic brain tumors using the apparent diffusion coefficient and normalized diffusion-weighted images. Magnetic Resonance Imaging, 21 (6): 645-650, 2003.

13- LAI P.H., HSU S.S., DING S.W., et al.: Proton magnetic resonance spectroscopy and diffusion-weighted imaging in intracranial cystic mass lesion. Surg. Neurol., 68: S25S36, 2007.

14- THAPA A., SUWAL S., CHATAUT D. and SUBEDI K. Role of Diffusion Weighted Imaging in Characterization of Intracranial Rim Enhancing Lesions. Nepalese Journal of Radiology, 9 (2): 17-23, 2020.

15- REICHE W., SCHUCHARDT V., HAGEN T., et al.: Differential diagnosis of intracranial ring enhancing cystic mass lesions-role of diffusion-weighted imaging (DWI) and diffusion-tensor imaging (DTI). Clin. Neurol. Neurosurg, 112 (3): 218-225, 2010.

16- HOLTAS S., GEIJER B., STROMBLAD L.G., et al.: A ring-enhancing metastasis with central high signal on diffusion-weighted imaging and low apparent diffusion coefficients. Neuroradiology, 42: 824-827, 2000.

17- WANG S., KIM S., CHAWLA S., et al.: Differentiation between Glioblastomas, Solitary Brain Metastases, and Primary Cerebral Lymphomas Using Diffusion Tensor and Dynamic Susceptibility Contrast-Enhanced MR Imaging. American Journal of Neuroradiology, 32 (3): pp. 507-514, 2011.

18- BHANDARI D., AGRAWAL D., TRIPATHI D., et al.: Role of Diffusion Weighted MRI in Intra-Axial Brain Tumours. Scholars Journal of Applied Medical Sciences, 4 (6): 2111-2119, 2016. 


\section{دور الرئين المغناطيسى بخاصية الانتشار للتميزبين الآفات الكيسية دوريسية داخل محورية الونية للدمارغ}

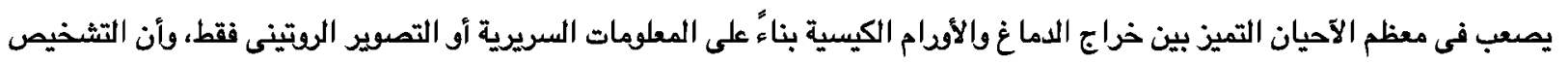

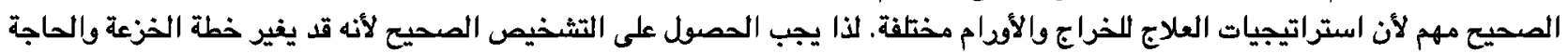
إلى تحاليل إضافية.

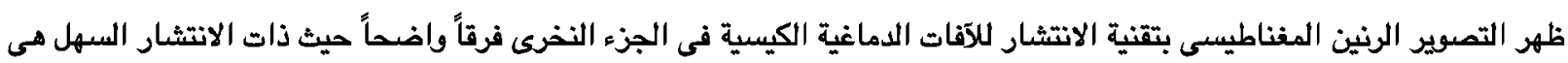

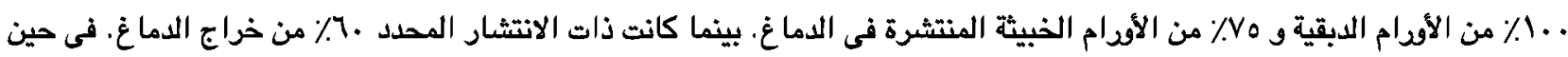

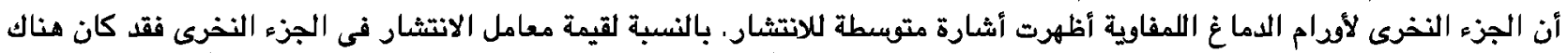

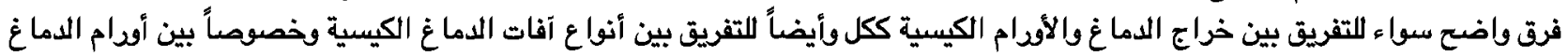
الدبقية والليمفاوية.

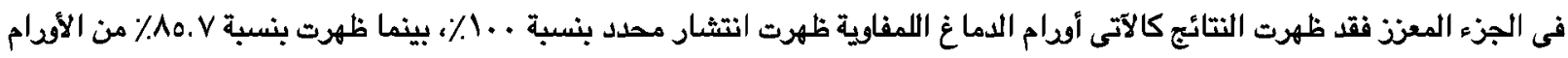

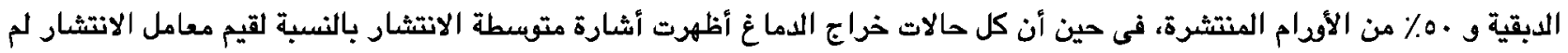

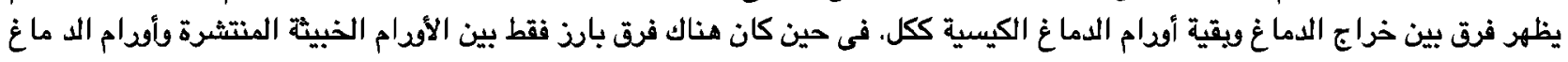

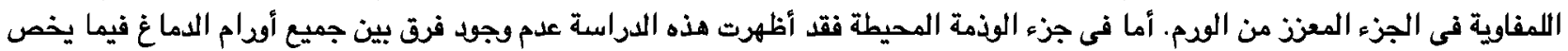
خاصية/لانتشار أو معامل الانتشار.

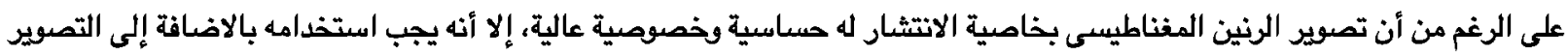

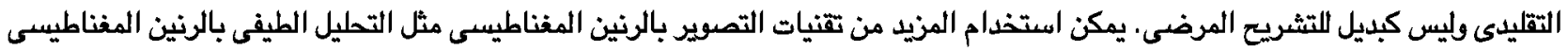

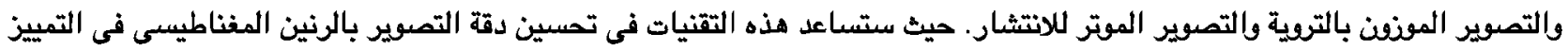
بين خراج الدماغ والأورام الكيسية أو النخرية. 